\title{
DO THE EFFECTIVE HEAT CONDUCTIVITY AND THE HEAT TRANSFER COEFFICIENT AT THE WALL INSIDE A PACKED BED DEPEND ON A CHEMICAL REACTION? WEAKNESSES AND APPLICABILITY OF CURRENT MODELS
}

\author{
R. J. WIJNGAARDEN and K. R. WESTERTERP \\ Chemical Reaction Engineering Laboratories, Department of Chemical Engineering, Twente University of \\ Technology, PO Box 217, 7500 AE Enschede, Netherlands
}

(First received 16 May 1988; accepted in revised form 15 December 1988)

\begin{abstract}
Many studies have been conducted on the effective heat conductivity $\left(\lambda_{\text {eff }}\right)$ and the heat transfer coefficient at the wall $\left(\alpha_{w}\right)$ inside packed beds. It has been mentioned that the values of $\lambda_{\text {eff }}$ and $\alpha_{w}$ are changed when a chemical reaction occurs in the packed bed. We give an explanation for such a phenomenon. The properties $\lambda_{\text {eff }}$ and $\alpha_{w}$ are lumped parameters which usually are determined by both the measured temperature profiles and the model used to calculate the temperature profiles from $\lambda_{\text {eff }}$ and $\alpha_{w}$. If either the experimental data are wrong or the model is erroneous the error will manifest itself in the values of $\lambda_{\text {eff }}$ and $\alpha_{w}$. At least a part of the change in the values of $\lambda_{\text {eff }}$ and $\alpha_{w}$ due to a chemical reaction is caused by the fact that a homogeneous model with catalyst and gas having the same temperature is chosen, whereas a heterogeneous model with catalyst and gas having different temperatures should be used. If no reaction occurs the catalyst and gas will have the same temperature and the homogeneous model yields a good description. Hence, when fitting temperature profiles with this model the correct values of $\lambda_{\text {eff }}$ and $\alpha_{w}$ are found. If reaction does occur the catalyst and the gas will have different temperatures because the heat of reaction must be transferred from the catalyst to the gas. If, despite this fact, a homogeneous model is used to calculate the temperature profiles, an error is introduced which is reflected in the values of $\lambda_{\text {eff }}$ and $\alpha_{w}$. As a consequence we create an apparent dependence of $\lambda_{\text {eff }}$ and $\alpha_{w}$ on the reaction rate. We derive criteria to determine which model must be used. We discuss results presented in the literature on the dependence of $\lambda_{\text {eff }}$ and $\alpha_{w}$ on the chemical reaction. The explanation is both qualitative and quantitative.
\end{abstract}

\section{INTRODUCTION}

Several studies have been conducted on the effective heat conductivity $\left(\lambda_{\text {eff }}\right)$ and the heat transfer coefficient at the wall $\left(\alpha_{w}\right)$ inside packed beds [see, for example, Damköhler (1937), Zehner (1973), Hennecke and Schlünder (1973), Lerou and Froment (1977), Schlünder (1966, 1978), Bauer (1977), Dixon and Cresswell (1979) and Hofmann (1979a, b)]. To this purpose models were developed for heat transfer inside packed beds. With these models the temperature profiles inside packed beds were fitted by varying $\lambda_{\text {eff }}$ and $\alpha_{w}$. Thus the values of $\lambda_{\text {eff }}$ and $\alpha_{w}$ obtained in the literature are best-fit values. Several authors reported that $\lambda_{\text {eff }}$ and $\alpha_{w}$ are dependent on a chemical reaction occurring inside the packed bed [see for example, Hofmann (1979b) and Chao et al. (1973)]. It is hardly likely that this dependence can be explained by experimental errors. It could also be possible that $\lambda_{\text {eff }}$ and $\alpha_{w}$ are affected by chemical reaction in some physico-chemical way. However, to our knowledge there is no indication whatsoever that can support this vision. The most probable explanation is that the model we use to fit $\lambda_{\text {eff }}$ and $\alpha_{w}$ yields wrong results when chemical reaction occurs. Since $\lambda_{\text {eff }}$ and $\alpha_{w}$ are fit parameters, an error in the model used will manifest itself in the values of $\lambda_{\text {eff }}$ and $\alpha_{w}$. In this paper we will give an example of this. This implicates that we must focus on the models that can be used to describe heat transfer in packed beds. A packed bed is usually described by deterministic models. These usually arise from mass and energy balances on a micro scale over an infinitesimally small control volume of the packed bed. There are a number of options for these models:

(1) It can be either one- or two-dimensional. Onedimensional means that only axial effects are taken into account; the two-dimensional models take both axial and radial effects into account.

(2) It can be either homogeneous or heterogeneous. The homogeneous models assume that properties of the gas and catalyst phase are the same; the heterogeneous ones take into account that both phases have different properties, e.g. different temperatures.

Generally a two-dimensional, homogeneous model is used to fit temperature profiles with $\lambda_{\text {eff }}$ and $\alpha_{w}$. The assumption that the stready state in a packed bed can be described with a homogeneous model is more probable if no reaction occurs, because in that case the catalyst temperature must equal the gas temperature. However, if a reaction does occur, this assumption becomes doubtful, even in the steady state. Typically for cooled tubular reactors the temperatures of the gas and the catalyst differ by a few degrees, whereas the radial temperature differences in the tubes are of the order of magnitude of $10-30^{\circ} \mathrm{C}$ : it can be expected that 
the temperature difference between gas and catalyst has quite an impact on the radial temperature profile inside the tubes.

If a heterogeneous model is used instead of a homogeneous model the dependence of $\lambda_{\text {eff }}$ and $\alpha_{w}$ on the chemical reaction can be explained as follows. If an exothermic reaction is carried out, the catalyst temperature will be higher than the gas temperature. Since the reaction rate increases with increasing temperature, the reaction rate-which is determined by the catalyst temperature-is higher than we would calculate on the basis of a homogeneous model, where we calculate the reaction rate on the basis of the lower gas temperature. Since the reaction rate is higher than we calculate on the basis of our model, our radial temperature profile will also be more pronounced than we calculate on the basis of the homogeneous model. We can correct for this by choosing a lower value for $\lambda_{\text {eff }}$ and $\alpha_{w}$, which also results in a more pronounced profile. Hence if we fit temperature profiles with the $\lambda_{\text {eff }}$ and $\alpha_{w}$ of a homogeneous model, an exothermic reaction induces an apparent decrease in $\lambda_{\text {eff }}$ and $\alpha_{w}$. In reverse, if we carry out an endothermic reaction, the catalyst temperature will be lower than the gas temperature. Therefore the values of the reaction rate are lower than we would expect on the basis of a homogeneous model. Thus the temperature profiles will be less pronounced than we expect, which corresponds to higher values of $\lambda_{\text {eff }}$ and $\alpha_{w}$. In other words, for endothermic reactions the effect on $\lambda_{\text {eff }}$ and $\alpha_{w}$ is reversed with respect to that for exothermic ones. We will quantify the explanation given above and will study to this end both a homogeneous and a heterogenous model. The models will be compared and characteristic parameters will be deduced. With those parameters we will derive criteria to determine whether or not a homogeneous model can be used, we will calculate the change in the values of $\lambda_{\text {eff }}$ and $\alpha_{w}$ if a chemical reaction occurs, and we will compare our results with experimental data given in the literature. To arrive at simple and understandable models we will make certain assumptions.

\section{ASSUMPTIONS}

The catalyst pellets in a packed bed have a very small contact spot area [see. Fig. 1 and Bauer (1977)]. Hence, if heat must be transported from one pellet to another, it must be transported via the gas phase. So heat transport from the solid catalyst phase to the gas and from the gas phase to other pellets or the wall can be regarded as two processes in series. Also a model could have been chosen where heat transfer takes place via the solid and gas phase in parallel. Then it is assumed that there is a significant contribution of the heat flow through the contact spot and that heat transfer between the solid and gas phase is negligible. Since the last assumption is obviously wrong, no parallel models have been presented in the literature. Combinations of the series and parallel model have been presented, among others, by Cresswell (1987). No

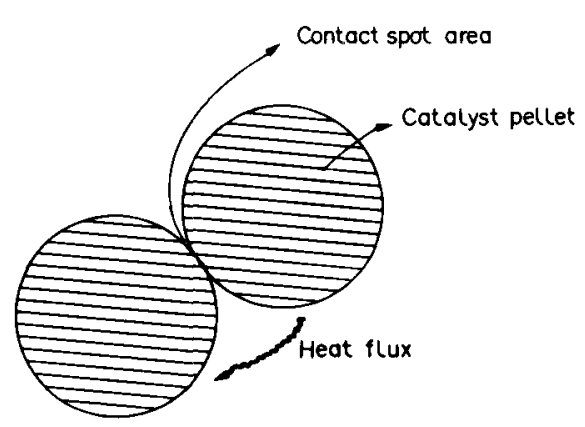

Fig. 1. Heat flow and contact spot area in packed beds.

experimental data have been obtained showing a significant heat flow via the contact spot area. We strongly feel that parallel heat flow can be neglected compared to the series flow. At this point, the line of reasoning would be detained too much if all reasons were discussed; therefore we elaborate on this subject in the last appendix of this paper, Appendix D. For heterogeneous models heat transport in series implies that the catalyst temperature is determined by the heat flux from the pellet to the gas $\left(\alpha_{p}\right)$, and the gas temperature is determined by the radial heat flux to the coolant $\left(\lambda_{\text {eff }}, \alpha_{w}\right)$. A consequence of the fact that the heat transport via the catalyst and gas phase occur in series is that if we shove a thermocouple in a packed bed we will measure the gas temperature, not the catalyst temperature. Since temperature profiles are usually measured this way, $\lambda_{\text {eff }}$ and $\alpha_{w}$ are determined from the gas temperature profiles. This becomes of importance if we discuss the heterogeneous model.

In order to derive a simple and understandable correlation furthermore we have made the following assumptions:

(1) in the micro heat balance heat transported by axial convection can be neglected compared to the amount of heat produced by chemical reaction,

(2) there are no radial concentration profiles,

(3) the reaction rate may be linearized with respect to the temperature,

(4) external mass transfer limitation between the pellet and the gas may be neglected,

(5) the physical properties do not depend on the temperature.

Hereunder we will elaborate on these assumptions and explain their usefulness and acceptibility:

(1) To envisage that the amount of heat transported by the forced convection in the axial direction is negligible compared to the amount of heat produced by chemical reaction, we consider a simple first-order reaction taking place in a plug flow reactor and with a kinetic constant independent of the temperature. This case is further elaborated in Appendix A and from that it follows that the following dimensionless group dominates the phenomenon: 


$$
\xi=\frac{N T U}{N R U}=\frac{4 U}{\rho_{g} C_{p, g} k D_{t}}
$$

being the ratio of the number of heat transfer units $(N T U)$ for heat transfer to the coolant divided by the number of reaction units $(N R U)$. The importance of the number $\xi$ is illustrated by the fact that the hot-spot temperature can be calculated directly from it, according to

$$
\frac{T_{h s}-T_{c}}{\Delta T_{\text {ad }}}=\xi^{\left(\frac{\xi}{1-\xi}\right)}
$$

(see Appendix A). Here $\Delta T_{\mathrm{ad}}$ is the adiabatic temperature rise in the feed gas:

$$
\Delta T_{\mathrm{ad}}=\frac{C_{\mathrm{in}}(-\Delta H)}{\rho_{g} C_{p, g}} .
$$

Equation (2) is illustrated in Fig. 2. Notice that, depending on the value of $\xi$, two regimes can be distinguished. For $\xi<0.1$ eq. (2) yields

$$
\frac{T_{h s}-T_{c}}{\Delta T_{\text {ad }}}=1 .
$$

Hence the hot-spot temperature equals the coolant temperature plus the adiabatic temperature rise. For $\xi>2$ eq. (2) yields

$$
\frac{T_{h s}-T_{c}}{\Delta T_{\mathrm{ad}}}=\frac{1}{\xi}=\frac{N R U}{N T U} .
$$

Thus the hot-spot temperature decreases if the $N R U$ decreases with respect to the $N T U$. For industrial adiabatic packed bed reactors $\xi$ roughly equals $10^{-3}-10^{-2}$ so in this case we operate in the low- $\xi$ regime. For industrial cooled tubular reactors $\xi$ roughly equals $10-100$ and we operate in the high- $\xi$ regime. For these reactors the difference between the hot-spot temperature and the coolant temperature is roughly $10-100$ times smaller than the adiabatic temperature rise.

From the discussion given in Appendix A it follows that heat transport by convection can be neglected always in the area around the hot spot. In the tail behind the hot-spot it can be neglected only if

$$
\xi>5 \text {. }
$$

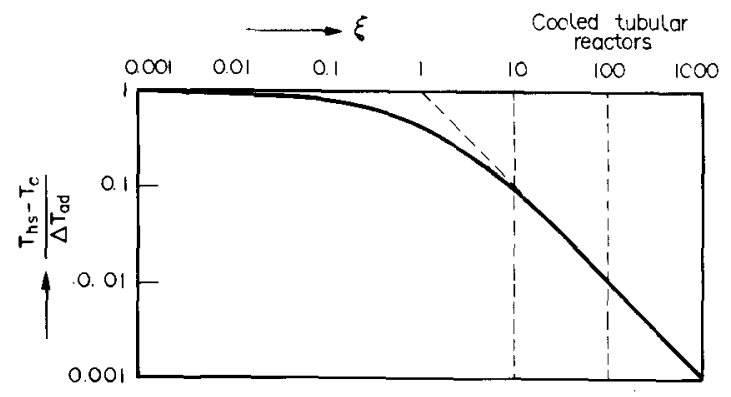

Fig. 2. Dimensionless hot-spot temperature $\left[\left(T_{h s}\right.\right.$ $\left.\left.-T_{c}\right) / \Delta T_{\mathrm{ad}}\right]$ vs the ratio $(\xi)$ of the number of transfer units $(N T U)$ to the number of reaction units $(N R U)$ for packed beds.
Therefore heat transport by convection can be neglected for cooled tubular reactors in and behind the hot-spot; for adiabatic packed bed reactors it can never be neglected. Obviously for adiabatic packed bed reactors we are not interested in the values of $\lambda_{\text {eff }}$ and $\alpha_{w}$ since almost no radial heat transport takes place anyway. Therefore we further focus on cooled tubular reactors and neglect the axial heat transport by convection.

(2) The radial concentration profiles in cooled tubular reactors are caused by the radial temperature profiles. In the centre of the bed the temperature has its highest value; thus the reaction rate has its highest value and consequently the concentration its lowest value. In Appendix B it is shown that the radial concentration profile can be neglected if

$$
\frac{R_{t}^{2} R\left(T_{c}\right)}{2 \mathscr{D}_{\mathrm{eff}} C_{b}} \frac{E_{a}}{R T_{c}} \frac{T_{m}-T_{c}}{T_{c}} \ll 1 .
$$

Inserting some typical values $-R_{t} \approx 0.05 \mathrm{~m}, \mathscr{D}_{\text {eff }} \approx$ $10^{-3} \mathrm{~m}^{2} / \mathrm{s}, R\left(T_{c}\right) / C_{b} \approx 0.1 \mathrm{~s}^{-1}, E_{a} / R T_{c} \approx 20$-we find that

$$
\theta_{m}=\frac{T_{m}-T_{c}}{T_{c}} \ll 0.4
$$

This gives an indication when the radial concentration gradient starts playing a role, which is hardly ever.

(3) To study the case where radial temperature gradients are small enough to allow linearization of the dependence of reaction rate on temperature, we assume the dependence of the reaction rate on the temperature to be of the Arrhenius type. In a secondorder approximation the reaction rate can be written as

$$
\begin{aligned}
R(T)= & R\left(T_{c}\right)+\left.\left(T-T_{c}\right) \frac{\partial R(T)}{\partial T}\right|_{T=T_{c}} \\
& +\left.\frac{1}{2}\left(T-T_{c}\right)^{2} \frac{\partial^{2} R(T)}{\partial T^{2}}\right|_{T=T_{c}} \\
= & R\left(T_{c}\right)\left[1+\left(\frac{E_{a}}{R T_{c}} \frac{T-T_{c}}{T_{c}}\right)\right. \\
& \left.+\frac{1}{2}\left(\frac{E_{a}}{R T_{c}} \frac{T-T_{c}}{T_{c}}\right)^{2}\left(1-2 \frac{R T_{c}}{E_{a}}\right)\right]
\end{aligned}
$$

Thus linearization is allowed if

$$
\frac{1}{2} \frac{E_{a}}{R T_{c}}\left|\frac{T-T_{c}}{T_{c}}\right|\left|1-2 \frac{R T_{c}}{E_{a}}\right| \ll 1 .
$$

Since the temperature is extreme in the centre of the packed bed, it is sufficient to demand that

$$
\left|\frac{T_{m}-T_{c}}{T_{c}}\right| \ll \frac{2}{\left|\left(E_{a} / R T_{c}\right)-2\right|}
$$

or

$$
\left|\theta_{m}\right| \ll \frac{2}{|\beta|}
$$


with

$$
\beta=\frac{E_{a}}{R T_{c}}-2 .
$$

Almost always $\beta$ is between 10 and 20 [see, for example, Table 1 taken from Hlavacek and Kubicek (1970)]. Hence requirement (12) is more stringent than requirement (8). Thus the linearization of the reaction rate is no longer allowed long before radial concentration gradients play a role. Therefore, we need not consider radial concentration gradients nor requirement (8).

(4) External mass transfer limitation may be neglected if

$$
\frac{C_{g}-C_{k}}{C_{g}} \ll 1 .
$$

A mass and heat balance for the catalyst pellet yields

$$
\begin{gathered}
R\left(C_{k}, T_{k}\right) V_{p}=k_{g} A_{p}\left(C_{g}-C_{k}\right) \\
R\left(C_{k}, T_{k}\right) V_{p}(-\Delta H)=\alpha_{p} A_{p}\left(T_{k}-T_{g}\right)
\end{gathered}
$$

It follows that

$$
\begin{aligned}
\frac{C_{g}-C_{k}}{C_{g}}= & \frac{\alpha_{p}}{k_{g}} \frac{T_{k}-T_{g}}{C_{g}(-\Delta H)}=\frac{\alpha_{p}}{\rho_{g} C_{p, g} k_{g}}\left(T_{k}-T_{g}\right) \\
& \times\left[\frac{\rho_{g} C_{p, g}}{C_{g}(-\Delta H)}\right]=\frac{\alpha_{p}}{\rho_{g} C_{p, g} k_{g}} \frac{T_{k}-T_{g}}{\Delta T_{\mathrm{ad}}} .
\end{aligned}
$$

We can write

$$
\frac{\alpha_{p}}{\rho_{g} C_{p, g} k_{g}}=\frac{N u}{S h} L e .
$$

For gases $L e \approx 1$ and hence, according to the Chilton-Colburn analogy, $N u / S h \approx 1$. Therefore we may write

$$
\frac{\alpha_{p}}{\rho_{g} C_{p, g} k_{g}} \approx 1
$$

and thus

$$
\frac{C_{g}-C_{k}}{C_{g}} \approx \frac{T_{k}-T_{g}}{\Delta T_{\mathrm{ad}}} \approx 10^{-2}-10^{-3}
$$

Hence we can conclude that in general external mass transfer limitation can be neglected.

(5) Compared to the reaction rate, physical properties depend only weakly on the temperature. Therefore in case the reaction rate can be linearized, without any

Table 1. Some typical values of $\beta$ for industrial processes [data taken from Hlavacek and Kubicek (1970)]

\begin{tabular}{lc}
\hline \multicolumn{1}{c}{ Process } & $\beta$ \\
\hline Ethylene hydrogenation & 11.2 \\
Benzene hydrogenation & 13.7 \\
Methane oxidation & 19.5 \\
Ethylene oxidation & 11.5 \\
Naphtalene oxidation & 20.2 \\
Acrylonitrile synthesis & 7.9 \\
\hline
\end{tabular}

doubt the assumption that the other physical and chemical properties are temperature-independent is correct. Hence adherence to criterion (12) is sufficient to guarantee that this assumption is valid. Summarizing it follows that the assumptions given above are correct if:

(1) we restrict ourselves to cooled tubular reactors,

(2) the following criterion is adhered to:

$$
\left|\beta \theta_{m}\right| \ll 2
$$

where $\theta_{m}$ is a measure for the temperature in the centre of the packed bed:

$$
\theta_{m}=\frac{T_{m}-T_{c}}{T_{c}}
$$

and $\beta$ is a measure for the dependence of the reaction rate on the temperature. If this dependence is of the Arrhenius type:

$$
\beta=\frac{E_{a}}{R T_{c}}-2
$$

or, more generally:

$$
\beta=\left.T_{c}\left[\left.\frac{\partial R(T)}{\partial T}\right|_{T=T_{c}}\right]^{-1} \frac{\partial^{2} R(T)}{\partial T^{2}}\right|_{T=T_{c}} .
$$

We further will assume these conditions to be fulfilled and apply them to the homogeneous and heterogeneous model.

\section{HOMOGENEOUS MODEL}

A heat balance on a micro scale, using the assumptions stated above, yields, for the homogeneous model:

$$
\frac{-\lambda_{\text {eff }}}{r} \frac{\partial}{\partial r}\left(r \frac{\partial T}{\partial r}\right)=R(T)(-\Delta H) .
$$

If we linearize the reaction rate:

$$
R(T)=R\left(T_{c}\right)\left[1+\left.\frac{T-T_{c}}{T_{c}} \frac{T_{c}}{R\left(T_{c}\right)} \frac{\partial R(T)}{\partial T}\right|_{T=T_{c}}\right]
$$

and introduce the following dimensionless numbers:

$$
\begin{aligned}
\rho & =\frac{r}{R_{t}} \\
\theta & =\frac{T-T_{\mathrm{c}}}{T_{c}} \\
\eta & =\left.\frac{T_{c}}{R\left(T_{c}\right)} \frac{\partial R(T)}{\partial T}\right|_{T=T_{c}} . \\
\gamma & =\frac{R\left(T_{c}\right)(-\Delta H) R_{t}^{2}}{\lambda_{\text {eff }} T_{c}} .
\end{aligned}
$$

Equation (23) can be written as

$$
-\frac{1}{\rho} \frac{\partial}{\partial \rho}\left(\rho \frac{\partial \theta}{\partial \rho}\right)=\gamma(1+\eta \theta) .
$$

This differential equation must be solved subject to the boundary conditions: 


$$
\begin{aligned}
& \rho=0 \Rightarrow \frac{\partial \theta}{\partial \rho}=0 \\
& \rho=1 \Rightarrow \frac{\partial \theta}{\partial \rho}=-B i \frac{R_{t}}{d_{p}} \theta
\end{aligned}
$$

with

$$
B i=\frac{\alpha_{w} d_{p}}{\lambda_{\text {eff }}} .
$$

The solution to this differential equation can be found as

$$
\theta=\frac{1}{\eta}\left[\frac{J_{0}(\sqrt{\eta \gamma} \rho)}{J_{0}(\sqrt{\eta \gamma})-\sqrt{\eta \gamma} J_{1}(\sqrt{\eta \gamma}) /\left(B i R_{t} / d_{p}\right)}-1\right]
$$

with $J_{0}$ and $J_{1}$ being Bessel functions of zeroth and first order, respectively. Some examples of temperature profiles obtained with eq. (33) are given in Fig. 3. This solution already serves our purposes; we now have to study the heterogeneous model.

\section{HETEROGENEOUS MODEL}

Under the assumptions stated before a heat balance on a micro scale for the gas phase in the heterogeneous model yields

$$
\frac{-\lambda_{\mathrm{eff}}}{r} \frac{\partial}{\partial r}\left(r \frac{\partial T_{g}}{\partial r}\right)=\alpha_{p}\left(T_{k}-T_{g}\right) \frac{A_{p}}{V_{p}}(1-\varepsilon)
$$

where $(1-\varepsilon) A_{p} / V_{p}$ represents the outer surface area of the catalyst per unit of reactor volume. The catalyst temperature $\left(T_{k}\right)$ follows from

$$
\alpha_{p} A_{p}\left(T_{k}-T_{g}\right)=V_{p} \frac{R\left(T_{k}\right)}{1-\varepsilon}(-\Delta H) .
$$

After linearization of $R\left(T_{k}\right)$ eq. (35) yields

$$
\begin{aligned}
& T_{k}-T_{g}= \frac{R\left(T_{c}\right)(-\Delta H) V_{p}}{(1-\varepsilon) \alpha_{p} A_{p}} \\
& \times\left[1+\left.\frac{T_{c}}{R\left(T_{c}\right)} \frac{\partial R(T)}{\partial T}\right|_{T=T_{c}} \frac{T_{k}-T_{c}}{T_{c}}\right] . \\
& \text { Вi }=2
\end{aligned}
$$

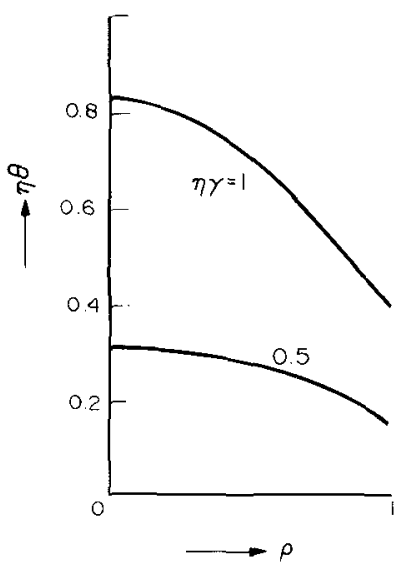

We introduce the following dimensionless numbers:

$$
\begin{aligned}
\theta_{g} & =\frac{T_{g}-T_{c}}{T_{c}} \\
\theta_{k} & =\frac{T_{k}-T_{c}}{T_{c}} \\
\delta= & \frac{R\left(T_{c}\right)(-\Delta H) V_{p}}{(1-\varepsilon) \alpha_{p} A_{p} T_{c}}=\frac{T_{k}-T_{g}}{T_{c}} \\
& \times\left[1+\left.\frac{T_{c}}{R\left(T_{c}\right)} \frac{\partial R(T)}{\partial T}\right|_{T=T_{c}} \frac{T_{k}-T_{c}}{T_{c}}\right]^{-1} .
\end{aligned}
$$

Hence the dimensionless group $\delta$ is a measure of the relative increase in the temperature due to the external heat resistance between the pellet and the gas. With $\eta$ and $\gamma$ given by eqs (27) and (28) we obtain

$$
\begin{gathered}
\frac{-1}{\rho} \frac{\partial}{\partial \rho}\left(\rho \frac{\partial \theta_{g}}{\partial \rho}\right)=\gamma\left(1+\eta \theta_{k}\right) \\
\theta_{k}-\theta_{g}=\delta\left(1+\eta \theta_{k}\right)
\end{gathered}
$$

subject to the boundary conditions (30) and (31). Substitution of eq. (41) in eq. (40) yields

$$
\begin{aligned}
\frac{-1}{\rho} \frac{\partial}{\partial \rho}\left(\rho \frac{\partial \theta_{g}}{\partial \rho}\right) & =\gamma\left[1+\eta\left(\frac{\theta_{g}}{1-\delta \eta}+\frac{\delta}{1-\delta \eta}\right)\right] \\
& =\frac{\gamma}{1-\delta \eta}\left(1+\eta \theta_{g}\right) .
\end{aligned}
$$

Comparing with eq. (29) we see that the same temperature profile is found with the homogeneous and the heterogeneous model, save for the fact that $\gamma$ in the homogeneous model must be replaced with $\gamma /(1-\delta \eta)$ to obtain the heterogeneous model. We will elaborate on this in the next section.

It is important to investigate when eq. (42) may be used. This is the case firstly if the assumptions stated before are applicable and secondly if the linearization used in eq. (36) is allowed. This linearization is allowed if

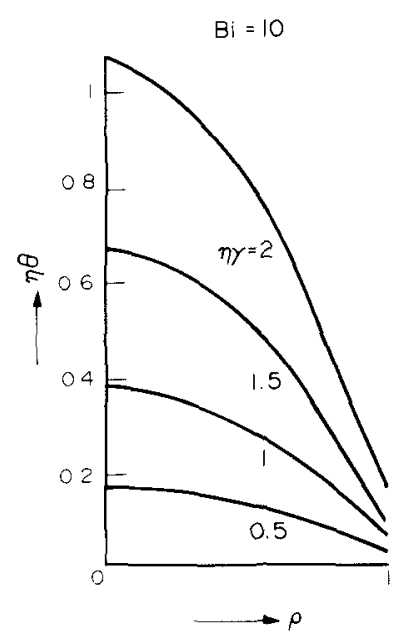

Fig. 3. Illustration of the radial temperature profiles, as found with the homogeneous model, for several values of $B i$ and $\sqrt{\eta \gamma}$. 


$$
\left|\frac{1}{2} \theta_{k} T_{c}\left[\left.\frac{\partial R(T)}{\partial T}\right|_{T=T_{c}}\right]^{-1} \frac{\partial^{2} R(T)}{\partial T^{2}}\right|_{T=T_{c}} \mid \ll 1 .
$$

Introduction of the number $\beta$, see eq. (22), gives

$$
\left|\beta \theta_{k}\right| \ll 2 \text {. }
$$

Substitution of eq. (41) yields

$$
\left|\frac{\beta}{1-\delta \eta}\left(\theta_{g}+\delta\right)\right| \ll 2 \text {. }
$$

Substituting some typical values like $\beta=10-20, \theta_{g}$ $=0.05-0.1$, and $\delta=0.01-0.02$ shows that this roughly may be written as

$$
\delta \eta \ll 0.5 \text {. }
$$

The formulae given in the next section can be used if the number $\delta \eta$ is smaller than 0.5 .

\section{COMPARISON OF THE HOMOGENEOUS AND THE HETEROGENEOUS MODEL}

It is convenient to define a dimensionless group $\zeta$ :

$$
\zeta=\delta \eta=\left.\frac{(-\Delta H) V_{p}}{(1-\varepsilon) \alpha_{p} A_{p}} \frac{\partial R(T)}{\partial T}\right|_{T=T_{c}} .
$$

The physico-chemical significance of $\zeta$ can be found by the following reasoning. After linearization of the reaction rate we can write

$$
\begin{aligned}
& T_{g}=T_{c}+\left[\left.\frac{\partial R(T)}{\partial T}\right|_{T=T_{c}}\right]^{-1}\left[R\left(T_{g}\right)-R\left(T_{c}\right)\right] \\
& T_{k}=T_{c}+\left[\left.\frac{\partial R(T)}{\partial T}\right|_{T=T_{c}}\right]^{-1}\left[R\left(T_{k}\right)-R\left(T_{c}\right)\right] .
\end{aligned}
$$

Substituting into eq. (35), it follows, for $\zeta$ :

$$
\zeta=\left.\frac{(-\Delta H) V_{p}}{(1-\varepsilon) \alpha_{p} A_{p}} \frac{\partial R(T)}{\partial T}\right|_{T=T_{c}}=\frac{R\left(T_{k}\right)-R\left(T_{g}\right)}{R\left(T_{k}\right)} .
$$

Hence the dimensionless number $\zeta$ is a measure of the relative increase in reaction rate due to the external heat resistance between the pellet and the gas. Therefore $\zeta$ is also a measure for the deviation between the homogeneous and the heterogeneous model. Alternatively $\zeta$ can be regarded as the ratio of the derivative at the coolant temperature of the pellet heat production rate and the derivative at the coolant temperature of the pellet heat withdrawal rate. The pellet heat production rate equals

$$
H P R=\frac{R(T)}{(1-\varepsilon)}(-\Delta H) V_{p}
$$

and hence

$$
\left.\frac{\partial H P R}{\partial T}\right|_{T=T_{c}}=\left.\frac{(-\Delta H) V_{p}}{(1-\varepsilon)} \frac{\partial R(T)}{\partial T}\right|_{T=T_{c}} .
$$

The pellet heat withdrawal rate equals

$$
H W R=\alpha_{p} A_{p}\left(T-T_{c}\right)
$$

and so

$$
\left.\frac{\partial H W R}{\partial T}\right|_{T=T_{c}}=\alpha_{p} A_{p} .
$$

Taking the ratio of eqs (52) and (54) it is found that $\zeta$ can also be written as

$$
\begin{aligned}
\zeta & =\left.\frac{(-\Delta H) V_{p}}{(1-\varepsilon) \alpha_{p} A_{p}} \frac{\partial R(T)}{\partial T}\right|_{T=T_{c}} \\
& =\left(\left.\frac{\partial H P R}{\partial T}\right|_{T=T_{c}}\right) /\left(\left.\frac{\partial H W R}{\partial T}\right|_{T=T_{c}}\right) .
\end{aligned}
$$

We want to call attention to the fact that we found the dimensionless group $(\partial H P R / \partial T) /(\partial H W R / \partial T)$ in several variations playing a dominant role in the description of the phenomena of heat transfer combined with chemical reaction [see Westerink and Westerterp (1988)].

As stated before the values of $\lambda_{\text {eff }}$ and $\alpha_{w}$ are determined by fitting experimental gas temperature profiles, not catalyst temperature profiles, with a homogeneous model. If we compare eqs (29) and (42) we see that, instead of determining a value $\gamma$ we think or hope we are determining, we actually obtain a value $\gamma^{*}$ equal to

$$
\gamma^{*}=\frac{\gamma}{1-\zeta}
$$

or instead of the actual value of $\lambda_{\text {eff }}$ we obtain a value $\lambda_{\text {eff }}^{*}$ :

$$
\begin{aligned}
\frac{R\left(T_{c}\right)(-\Delta H) R_{t}^{2}}{\lambda_{\text {eff }}^{*} T_{c}}= & \frac{R\left(T_{c}\right)(-\Delta H) R_{t}^{2}}{\lambda_{\text {eff }} T_{c}} \\
& \times \frac{1}{1-\zeta} \Leftrightarrow \lambda_{\text {eff }}^{*}=\lambda_{\text {eff }}(1-\zeta) .
\end{aligned}
$$

Since we determine the correct value of the Biot number - the boundary conditions remain the same-and, since $B i$ is proportional to $\lambda_{\text {eff }} / \alpha_{w}$, a change in the value of $\lambda_{\text {eff }}$ will automatically induce a change in the value of $\alpha_{w}$ :

$$
B i=\frac{\alpha_{w} R_{t}}{\lambda_{\text {eff }}}=B i^{*}=\frac{\alpha_{w}^{*} R_{t}}{\lambda_{\text {eff }}^{*}} .
$$

Thus also the value of $\alpha_{w}^{*}$ found by fitting experimental temperature profiles and the actual value of $\alpha_{w}$ differ according to

$$
\alpha_{w}^{*}=\alpha_{w}(1-\zeta)
$$

Notice that eqs (57) and (59) for exothermic reactions predict values of $\lambda_{\text {eff }}^{*}$ and $\alpha_{w}^{*}$ lower than the real values of $\lambda_{\text {eff }}$ and $\alpha_{w}$, and for endothermic reactions values higher than the real $\lambda_{\text {eff }}$ and $\alpha_{w}$. This is in agreement with the physical explanation given in the Introduction: for exothermic reactions the catalyst temperature is higher than the gas temperature, so the reaction rate is higher than expected and thus temperature profiles are more pronounced than expected, for which we have to correct by chosing lower values for $\lambda_{\text {eff }}$ and $\alpha_{w}$; 
for endothermic reactions according to the same reasoning we will find higher values for $\lambda_{\text {eff }}$ and $\alpha_{w}$.

We would like to emphasize that the formulae given here-hence also eqs (57) and (59)-can be used only if

$$
\zeta=\delta \eta<0.5
$$

because of the linearization we introduced. In order to further support the statement that the apparent dependence of $\lambda_{\text {eff }}$ and $\alpha_{w}$ on chemical reaction is highly significant, we note that Hofmann (1979b) reports that the number $\zeta$ should roughly equal $0.2-0.3$. However, he finds that values of $\lambda_{\text {eff }}$ and $\alpha_{w}$ are higher for exothermic reactions, not lower as predicted by us. This can be expected, since it is hardly likely that the correlations used to calculate $\lambda_{\text {eff }}$ and $\alpha_{w}$ without chemical reaction are very accurate. This is illustrated in Fig. 4, where $\lambda_{\text {eff }}$ and $\alpha_{w}$ are plotted vs the Peclet number for the case where there is no chemical reaction (for definition of $P e$ see Notation). The straight lines were calculated from the correlations given by Zehner (1973) and Dixon and Cresswell (1979); the dots indicate the values measured by Hofmann (1979b). Notice that there is a $25-80 \%$ deviation between the literature correlations and the values measured by Hofmann (1979b). Since a $10 \%$ error in those correlations will induce a much larger error in the value calculated for $\zeta$ [see eqs (57) and (59)], a considerable spread in the values for $\zeta$ must be expected.

Secondly, it must be mentioned that $\zeta$ depends on the velocity, not only because $\alpha_{p}$ depends on the velocity, but also because the axial concentration
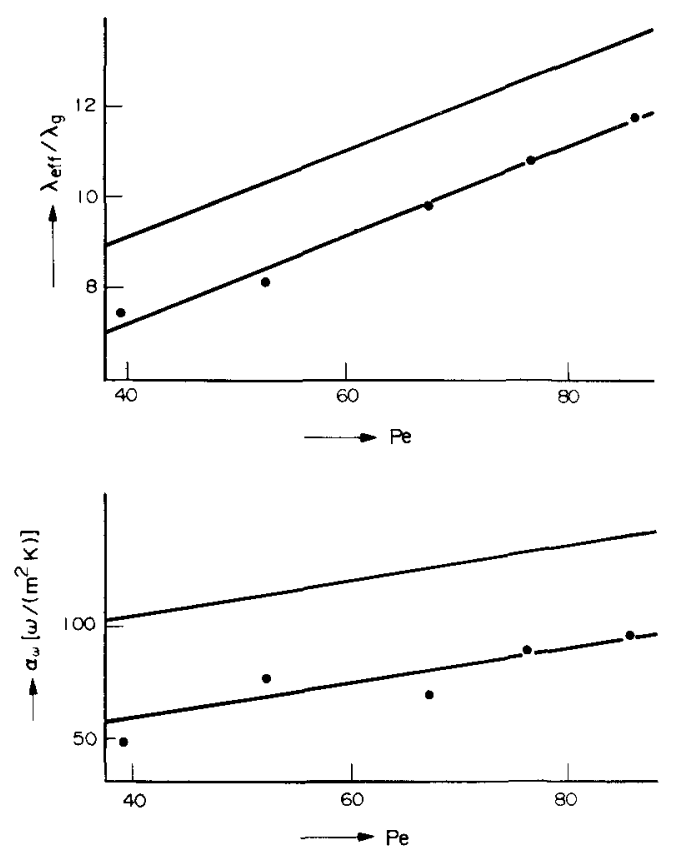

Fig. 4. Effective radial heat conductivity $\left(\lambda_{\text {eff }}\right)$ and the heat transfer coefficient at the wall $\left(\alpha_{w}\right)$ vs the superficial gas velocity for the no chemical reaction case. Straight lines obtained from literature correlations, dots measured by Hofmann (1979b). profile of the reactant is determined by the velocity. Since the reaction rate depends on the concentration, the derivative of the reaction rate with respect to the temperature depends on the concentration and therefore also $\zeta$ depends on the concentration. Thus $\zeta$ varies in the axial direction of the tube, or, if we calculate $\lambda_{\text {eff }}$ and $\alpha_{w}$ by fitting the radial temperature profile measured at one specific location in the axis of the tube, it depends on the velocity what the local value of $\zeta$ is in that particular place. Since we do not know where Hofmann $(1979 \mathrm{~b})$ measured his radial temperature profiles we cannot give exact data for the values to be expected for $\zeta$ in his experiments.

In Appendix $\mathrm{C}$ an order of magnitude is estimated for the number $\zeta$. The result is

$$
|\zeta|=0.01-0.6
$$

Now we easily derive a criterion to determine whether or not a homogeneous model can be used instead of a heterogeneous one. If we allow a $10 \%$ error then according to the eqs $(50),(57)$ and (59) a homogeneous model can be taken if

$$
|\zeta|<0.1
$$

This condition is fulfilled in the case when the reaction rate is low or hardly depends on the temperature or if the heat transfer between the catalyst pellet and the gas phase is very good.

\section{CONCLUSIONS AND SUMMARY}

A homogeneous model may be used instead of a heterogenous model if

$$
\begin{aligned}
|\zeta| & =\left.\frac{|-\Delta H| V_{p}}{(1-\varepsilon) \alpha_{p} A_{p}} \frac{\partial R(T)}{\partial T}\right|_{T=T_{c}}=\frac{\left|R\left(T_{k}\right)-R\left(T_{g}\right)\right|}{R\left(T_{k}\right)} \\
& =\left|\left(\left.\frac{\partial H P R}{\partial T}\right|_{T=T_{c}}\right) /\left(\left.\frac{\partial H W R}{\partial T}\right|_{T=T_{c}}\right)\right|=<0.1
\end{aligned}
$$

i.e. when the relative increase in reaction rate due to the external heat resistance between the pellet and the gas is smaller than $10 \%$.

If $|\zeta|>0.1$ a heterogeneous model should be used. If for that case we nevertheless use a homogeneous model the heterogeneous character will be lumped in the values of $\lambda_{\text {eff }}$ and $\alpha_{w}$. These values will increase for endothermic reactions $(\zeta<0)$ and decrease for exothermic reactions $(\zeta>0)$. We have shown that the apparent change in $\lambda_{\text {eff }}$ and $\alpha_{w}$ can be calculated from:

$$
\begin{gathered}
\lambda_{\text {eff }}^{*}=\lambda_{\text {eff }}(1-\zeta) \\
\alpha_{w}^{*}=\alpha_{w}(1-\zeta)
\end{gathered}
$$

which can be used for $\zeta<0.5$.

Although the assumptions for the models employed by us are rather crude, we believe that the dimensionless group $\zeta$ is the characteristic parameter for comparing the homogeneous and heterogeneous model. Also we feel there is no reason to assume that the values of $\lambda_{\text {eff }}$ and $\alpha_{w}$ are changed when a chemical reaction occurs. We are convinced that the system 
properties remain constant and are not influenced by a chemical reaction.

Acknowledgements - The authors wish to thank the Dutch Research Foundation SON and the Foundation for Technical Sciences STW for their financial support.

\section{NOTATION}

$a_{g} \quad$ thermal diffusity of the gas, $\mathrm{m}^{2} / \mathrm{s}$

$A_{p} \quad$ external surface area of the catalyst pellet, $\mathrm{m}^{2}$

$B i=\alpha_{w} d_{p} / \lambda_{\text {eff }}$, Biot number based on particle diameter

$C_{b} \quad$ bulk concentration of the reactant, $\mathrm{mol} / \mathrm{m}^{3}$

$C_{g}$ concentration of the reactant in the gas phase, $\mathrm{mol} / \mathrm{m}^{3}$

$C_{\text {in }} \quad$ inlet concentration of the reactant, $\mathrm{mol} / \mathrm{m}^{3}$

$C_{k} \quad$ concentration of the reactant in the internal catalyst pores, $\mathrm{mol} / \mathrm{m}^{3}$

$C_{p} \quad$ concentration of the reactant in the internal catalyst pores, $\mathrm{mol} / \mathrm{m}^{3}$

$C_{p, g} \quad$ specific heat of the gas, $\mathrm{J} /(\mathrm{kg} \mathrm{K})$

$d_{p} \quad=6 V_{p} / A_{p}$, particle diameter equivalent to a sphere, $\mathrm{m}$

$\mathscr{D}_{\text {eff }} \quad$ radial mass dispersion coefficient, $\mathrm{m}^{2} / \mathrm{s}$

$\mathscr{D}_{g}$ diffusion coefficient of the reactant in the gas phase, $\mathrm{m}^{2} / \mathrm{s}$

$E_{a} \quad$ energy of activation, $\mathrm{J} / \mathrm{mo}$

$H P R$ heat production rate, $\mathrm{W}$

$H W R$ heat withdrawal rate, $\mathrm{W}$

$(-\Delta H)$ reaction enthalpy, $\mathrm{J} / \mathrm{mol}$ reactant converted: for exothermic reactions $(-\Delta H)>0$; for endothermic reactions $(-\Delta H)<0$ reaction rate constant based on the catalyst volume, $s^{-1}$ mass transfer coefficient of the reactant between the pellet and the gas, $\mathrm{m} / \mathrm{s}$

Le $\quad=a_{g} / \mathscr{D}_{g}$, Lewis number

$L_{t} \quad$ tube length, length of the packed bed, $m$

$N R U=k L_{t} / v_{0}$, number of reaction units

$N T U=4 U L_{t} /\left(\rho_{g} C_{p, g} v_{0} D_{t}\right)$, number of heat transfer units

$\mathrm{Nu} \quad=\alpha_{p} d_{p} / \lambda_{g}$, particle Nusselt number

$\mathrm{Pe} \quad=v_{0} d_{p} / a_{g}$, particle Peclet number

$r$ radial coordinate, $m$

$R \quad$ gas constant, $8.314 \mathrm{~J} /(\mathrm{mol} \mathrm{K})$

$R(T)$ reaction rate at the temperature $T$ $[R(T)>0]$, mol reactant converted $/\left(\mathrm{m}^{3}\right.$ packed bed volume s)

$R_{t} \quad$ tube radius, $\mathrm{m}$

$T$ temperature, $\mathrm{K}$

$T_{c} \quad$ coolant temperature, $\mathrm{K}$

$T_{g} \quad$ gas temperature, $\mathrm{K}$

$T_{k} \quad$ catalyst temperature, $\mathrm{K}$

$T_{m} \quad$ temperature in packed bed centre, $\mathrm{K}$

$\Delta T_{\text {ad }} \quad=C_{g}(-\Delta H) /\left(\rho_{g} C_{p, g}\right)$, adiabatic temperature rise of the gas, $K$

$U$ overall heat transfer coefficient between the packed bed and the wall, $\mathrm{W} /\left(\mathrm{m}^{2} \mathrm{~K}\right)$

$v_{0} \quad$ superficial gas velocity based on the empty tube, $\mathrm{m} / \mathrm{s}$
$V_{p} \quad$ volume of a catalyst pellet, $\mathrm{m}^{3}$

$z \quad$ axial coordinate, $\mathrm{m}$

\section{Greek letters}

$\alpha_{p} \quad$ heat transfer coefficient between the particle and the gas, $W /\left(\mathrm{m}^{2} \mathrm{~K}\right)$

$\alpha_{w} \quad$ heat transfer coefficient between the packed bed and the wall, $\mathrm{W} /\left(\mathrm{m}^{2} \mathrm{~K}\right)$

$\alpha_{w}^{*} \quad$ apparent value of $\alpha_{w}$ when chemical reaction occurs, $\mathrm{W} /\left(\mathrm{m}^{2} \mathrm{~K}\right)$

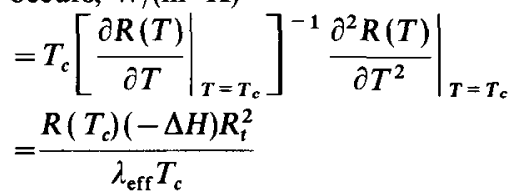

apparent value of $\gamma$ when chemical reaction occurs

$$
=\frac{R\left(T_{c}\right)(-\Delta H) V_{p}}{(1-\varepsilon) \alpha_{p} A_{p} T_{c}}
$$

porosity, volume fraction of the gas in the bed

$=\left.\frac{(-\Delta H) V_{p}}{(1-\varepsilon) \alpha_{\mathrm{p}} A_{\mathrm{p}}} \frac{\partial R(T)}{\partial T}\right|_{T=T_{c}}=\frac{R\left(T_{k}\right)-R\left(T_{g}\right)}{R\left(T_{k}\right)}$

$=\left(\left.\frac{\partial H P R}{\partial T}\right|_{T=T_{c}}\right) /\left(\left.\frac{\partial H W R}{\partial T}\right|_{T=T_{c}}\right)$

$\eta \quad=\left.\frac{T_{c}}{R\left(T_{c}\right)} \frac{\partial R(T)}{\partial T}\right|_{T=T_{c}}$

$\theta \quad=\left(T-T_{c}\right) / T_{c}$, dimensionless temperature

$\theta_{g} \quad=\left(T_{g}-T_{c}\right) / T_{c}$, dimensionless gas temperature

$\theta_{k}=\left(T_{k}-T_{c}\right) / T_{c}$, dimensionless catalyst temperature

$\theta_{m} \quad=\left(T_{m}-\dot{T}_{c}\right) / T_{c}$, dimensionless temperature of packed bed centre

$\lambda_{\text {eff }}$ effective radial heat conductivity of the packed bed, W/(m K)

$\lambda_{\text {eff }}^{*} \quad$ apparent value of $\lambda_{\text {eff }}$ when chemical reaction occurs, $\mathrm{W} /(\mathrm{m} \mathrm{K})$

kinematic viscosity of the gas, $\mathrm{m}^{2} / \mathrm{s}$ $=4 U /\left(\rho_{g} C_{p, g} k D_{t}\right)$, ratio of the number of heat transfer units and the number of reaction units

$\rho \quad=r / R_{t}$, dimensionless radial coordinate

$\rho_{g} \quad$ density of the gas, $\mathrm{kg} / \mathrm{m}^{3}$

$\omega \quad=z / L_{t}$, dimensionless axial coordinate

\section{REFERENCES}

Bauer, R., 1977, Effektive radiale Wärmeleitfähigkeit gasdürchströmter Schüttungen mit Partikeln unterschiedlicher Form und Grobenverteiling. VDI ForschHft. 582.

Chao, R. E., Cabon, R. A. and Irrizarry, M. M., 1973, Wall heat transfer to chemical reactors. Can. J. chem. Engng 51,

67.
Damköhler, G., 1937, Der Chemie-Ingenieur, Bd III, 1. Teil. Akademie, Leipzig.

Dixon, A. G. and Cresswell, D. L., 1979, Theoretical prediction of effective heat transfer parameters in packed beds. A.I.Ch.E. J. 25, 663.

Hennecke, F. W. and Schlünder, E. U., 1973, Wärmeübergang in beheizten oder gekühlten Rohren mit Schüttungen 
aus Kugeln, Zylindern und Raschig-Ringen. Chemie-IngrTech. 45, 277.

Hlavácek, V. and Kubicek, M., 1970, Modelling of chemical reactors-XXI. Effect of simultaneous heat and mass transfer inside and outside of a pellet on reaction--I. Chem. Engng. Sci. 25, 1537.

Hofmann, H., 1979a, Fortschritte bei der Modellierung von Festbettreaktoren. VDI-Ber. 349, 119.

Hofmann, H. 1979b, Fortschritte bei der Modellierung von Festbettreaktoren. Chemie-Ingr-Tech. 51, 257.

Lerou, J. J. and Froment, G. F., 1978, Estimation of heat transfer parameters in packed beds from radial temperature profiles. Chem. Engng J. 15, 233.

Schlünder, E. U., 1966, Wärme- und Stoffübertragung zwischen durchströmten Schüttungen und darin eingebetteten Einzelkörpern. Chemie-Ingr-Tech. 38, 967.

Schlünder, E. U., 1978, Transport phenomena in packed bed reactors. Chem. Reaction Engng Rev.-Houston ACS Symp. Ser. 72, 110.

Westerink, E. J. and Westerterp, K. R., 1988, Safe design of cooled tubular reactors for exothermic multiple reactions: multiple-reaction networks. Chem. Engng Sci. 43, 1051.

Zehner, P., 1973, Experimentelle und theoretische Bestimmung der effektiven Wärmeleitfähigkeit bei mäßigen und hohen Temperaturen. VDI ForschHft. 558.

\section{APPENDICES}

Appendix A: the contribution of the convective term in the micro heat balance according to the one-dimensional model, neglecting the temperature dependence of the reaction rate

To make a rough estimate as to whether the convective term in the heat balance can be neglected or not, we consider a first-order reaction taking place in a one-dimensional plug flow reactor and with a reaction rate independent of temperature. For micro balances for mass and heat yield (see Fig. A1):

$$
\begin{aligned}
& \frac{\partial \phi}{\partial \omega}=-N R U \phi \\
& \frac{\partial \theta}{\partial \omega}=N R U \Delta \theta_{\mathrm{ad}} \phi-N T U \theta
\end{aligned}
$$

where we introduced the parameters:

$$
\begin{gathered}
\omega=\frac{z}{L_{t}} \\
\phi=\frac{C}{C_{\text {in }}}
\end{gathered}
$$

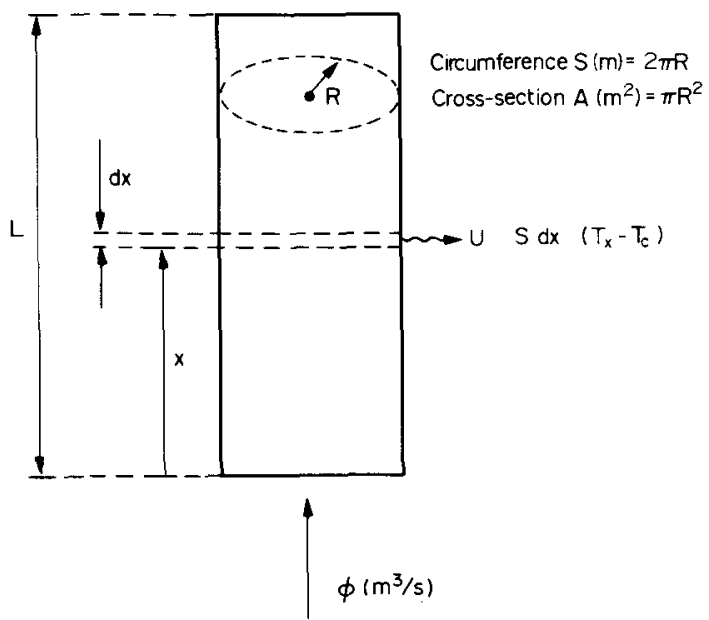

Fig. A1. Infinitesimally small volume element $(A \mathrm{~d} x)$ in a plug flow reactor.

$$
\begin{aligned}
\Delta \theta_{\mathrm{ad}} & =\frac{C_{\mathrm{in}}(-\Delta H)}{\rho_{g} C_{p, g} T_{c}} \\
N T U & =\frac{4 L_{t} U}{\rho_{g} C_{p, g} v_{0} D_{t}} \\
N R U & =\frac{k L_{t}}{v_{0}}
\end{aligned}
$$

and $\theta$ as given in the Notation. If the gas inlet temperature equals the coolant temperature, which is very often true for cooled tubular reactors, this set of equations must be solved subject to the initial conditions:

$$
\omega=0 \Rightarrow \theta=0 \wedge \phi=1 \text {. }
$$

From this we find that

$$
\theta=N R U \Delta \theta_{\mathrm{ad}} \frac{e^{-N T U \omega}-e^{-N R U \omega}}{N R U-N T U} .
$$

According to this model a hot-spot occurs for

$$
\frac{\partial \theta}{\partial \omega}=0 \Rightarrow \omega_{h s}=\frac{\ln (N R U / N T U)}{N R U-N T U} .
$$

The hot-spot will be located inside the reactor if $\omega_{h s}<1$, so if

$$
\frac{\ln (N R U / N T U)}{N R U-N T U}<1 .
$$

This is illustrated in Fig. A2. Notice that a hot-spot in the packed bed is favoured by both high $N T U$ values and high $N R U$ values.

The hot-spot temperature can be found by substitution of eq. (A10) in eq. (A11), which yields

$$
\frac{\theta_{h s}}{\Delta \theta_{\text {ad }}}=\frac{T_{h s}-T_{c}}{\Delta T_{\text {ad }}}=\xi^{\left(\frac{\xi}{1-\xi}\right) .}
$$

This is illustrated in Fig. 2 .

Heat transported by convection in the axial direction can be neglected as compared to the amount of heat produced by chemical reaction if [see eq. (A2)]

$$
\left|\frac{\partial \theta}{\partial \omega}\right| \ll N R U \Delta \theta_{\mathrm{ad}} \phi
$$

According to eq. (A9) this condition can be written as

$$
\left|\frac{N R U-N T U e^{(N R U-N T U) \omega}}{N R U-N T U}\right| \ll 1
$$

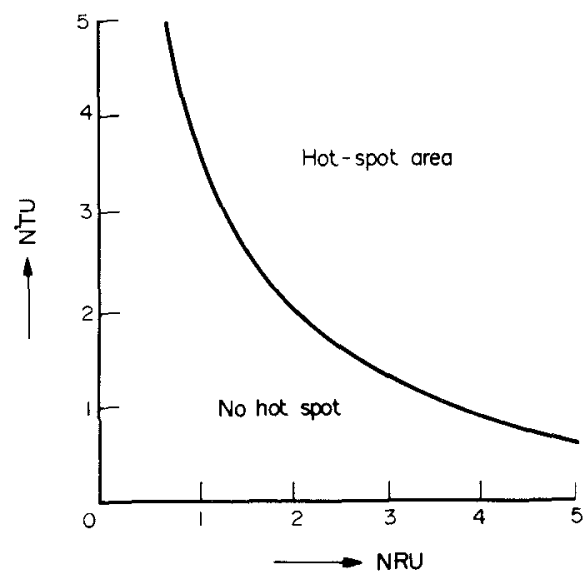

Fig. A2. Values of $N T U$ and $N R U$ where a hot-spot region in packed-bed reactors can be expected. 
or, since

$$
\omega_{h s}=\frac{\ln (N R U / N T U)}{N R U-N T U}
$$

eq. (A14) can also be written as

$$
\left|\frac{1-\xi^{\left(\frac{\omega_{h s}-\omega}{\omega_{h s}}\right)}}{1-\xi}\right| \ll 1 .
$$

This is illustrated in Fig. A3 It will be clear that condition (A13) holds:

(1) always near the hot-spot;

(2) and also before and behind the hot-spot if $\xi$ is large, say larger than 5 . Consequently for cooled tubular reactors with large $\xi$ values, typically $\xi=10-100$, the neglect of axial convection in the micro heat balance is a very reasonable assumption. This assumption will pose some problems only very close to the reactor entrance; for the remainder of the reactor tube length it holds.

On the other hand for industrial adiabatic packed bed reactors $\xi$ values are very low, typically $\xi=10^{-2}-10^{-3}$ : typically $N R U=1-10$ and $N T U=10^{-2}-10^{-3}$, so according to eq. (A11) we operate outside the hot-spot region (see Fig. A2). Hence for adiabatic bed reactors $\xi$ is very low and $\omega / \omega_{h s} \ll 1$. As a consequence, from Fig. A3 it can be seen that for these types of reactors the convection term in the heat balance cannot be neglected anymore, since $|\partial \theta / \partial \omega| /\left(N R U \Delta \theta_{\text {ad }} \phi\right)$, which is shown in Fig. A3 vs $\omega / \omega_{h s}$, remains close to $\left(\omega / \omega_{h s} \ll 1\right)$.

Summarizing it may be concluded that axial convection of heat indeed can be neglected as long as we refer to cooled tubular reactors only.

Appendix B: when can we neglect radial concentration profiles? A rough estimation

Consider a packed bed between two infinitely long flat slabs (see Fig. B1). Between the two slabs there is a linear temperature profile. The temperature gradient is very small, so that the reaction rate can be linearized. Hence there will also be a linear reaction rate profile (see Fig. B1).

We can calculate the maximum driving force for radial dispersion of the reactant by assuming that the reaction rate on the left-hand side of the catalyst bed is $\boldsymbol{R}_{c}$, whereas it is $\boldsymbol{R}_{h}$ on the right-hand side If the cross-sectional area parallel to the two slab walls of the bed is $A\left(\mathrm{~m}^{2}\right)$, the difference between the amount of reactant converted on the left-hand side and the right-hand side is

$$
\left(R_{h}-R_{c}\right) \frac{1}{2} R A(\mathrm{~mol} / \mathrm{s})
$$

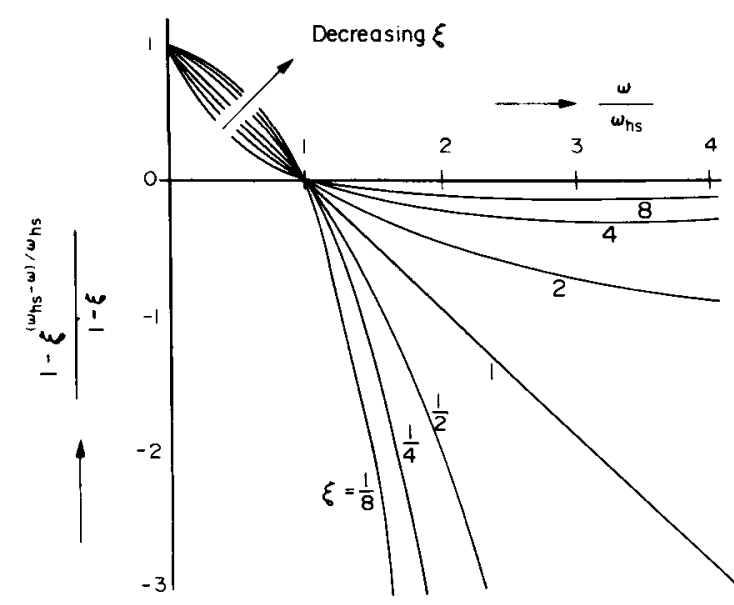

Fig. A3. Relative contribution of axial convection vs an axial coordinate $\left(\omega / \omega_{h s}\right)$.

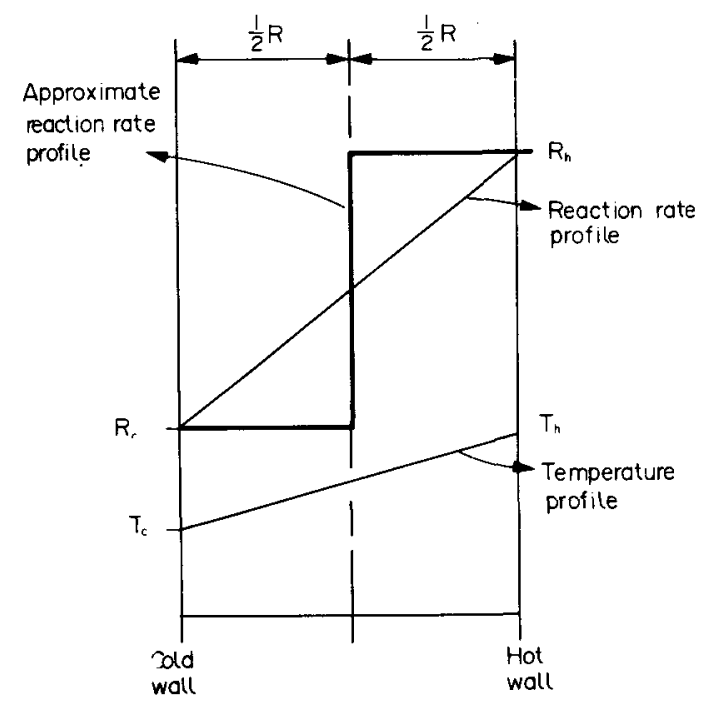

Fig. B1. Illustration of temperature and reaction rate profiles inside a bed packed between two infinite slabs.

If the difference of the concentrations at the hot and cold wall equals $\Delta C=C_{c}-C_{b}$, the flux through the centre plane roughly is equal to

$$
\frac{\Delta C}{R} A \mathscr{D}_{\text {eff }}(\mathrm{mol} / \mathrm{s})
$$

Hence the maximum concentration difference is roughly given by

$$
\Delta C=\frac{R^{2}}{2 \mathscr{D}_{\mathrm{eff}}}\left(R_{h}-R_{\mathrm{c}}\right) \approx \frac{R^{2}}{2 \mathscr{D}_{\mathrm{eff}}}\left(T_{h}-T_{c}\right) \times\left.\frac{\partial R(T)}{\partial T}\right|_{T=T_{c}} .
$$

If the dependence of the reaction rate on the temperature is of the Arrhenius type, this can be written as

$$
\Delta C=\frac{R^{2} R\left(T_{c}\right)}{2 \mathscr{D}_{\mathrm{eff}}} \frac{E_{a}}{R T_{c}} \frac{T_{h}-T_{c}}{T_{c}}
$$

or, if the average concentration in the bed equals $C_{b}$, the relative concentration difference is given by

$$
\frac{\Delta C}{C_{b}}=\frac{R^{2} R\left(T_{c}\right)}{2 \mathscr{D}_{\mathrm{eff}} C_{b}} \frac{E_{a}}{R T_{c}} \frac{T_{h}-T_{c}}{T_{\mathrm{c}}} .
$$

Hence the concentration gradient across the bed can be neglected if

$$
\frac{R^{2} R\left(T_{c}\right)}{2 \mathscr{D}_{\mathrm{eff}} C_{b}} \frac{E_{a}}{R T_{c}} \frac{T_{h}-T_{c}}{T_{c}} \ll 1
$$

Appendix $C$ : an estimation of the order of magnitude of the number $\zeta$ which is a measure for the heterogeneity of a packed bed with chemical reaction

Here we will make an estimation for the number $\zeta$. For that we will use the definition

$$
\zeta=\left.\frac{(-\Delta H) V_{p}}{(1-\varepsilon) \alpha_{p} A_{p}} \frac{\partial R(T)}{\partial T}\right|_{T=T_{c}} .
$$

Roughly the following figures may be used for industrial reactors:

$$
\begin{aligned}
& |-\Delta H| \approx 500 \times 10^{3} \mathrm{~J} / \mathrm{mol} \text { reactant } \\
& \varepsilon \approx 0.5 \\
& \alpha_{p} \approx 100 \mathrm{~W} /\left(\mathrm{m}^{2} \mathrm{~K}\right) \\
& V_{p} / A_{p} \approx 10^{-3} \mathrm{~m} .
\end{aligned}
$$


If the dependence of the reaction rate on the temperature is of the Arrhenius type we may write

$$
\left.\frac{\partial R(T)}{\partial T}\right|_{T=T_{c}}=\frac{E_{a}}{R T_{c}} \frac{R\left(T_{c}\right)}{T_{c}} .
$$

Typical reaction rates range from $0.1 \mathrm{~mol}$ reactant $/\left(\mathrm{m}^{3}\right.$ bed $\left.\mathrm{s}\right)$ up to $1 \mathrm{~mol}$ reactant $/\left(\mathrm{m}^{3}\right.$ bed s): further $E_{a} /\left(R T_{c}\right)=10-20$ (see Table 1), and $T_{c}=300-800 \mathrm{~K}$, so that

$$
\left.\frac{\partial R(T)}{\partial T}\right|_{T=T_{c}}=0.01-0.6 \mathrm{~mol} \text { reactant } /\left(\mathrm{m}^{3} \text { bed } \mathrm{s}\right) .
$$

Inserting the values obtained into eq. $(\mathrm{C} 1)$ we find that

$$
|\zeta|=0.01-0.6 \text {. }
$$

Appendix D: heat transport inside packed beds. Arguments for the series model

We assume a series model for heat transport in the bed in which heat transfer between two pellets via their contact spot area is neglected compared to the amount of heat transported by the gas phase near the pellet contact points and around the pellet. In this series model we have heat transfer from the solid phase to the gas phase and radial heat transfer within the gas phase to the wall. To a certain extent this choice is a rather arbitrary one: all heat transport properties in a packed bed are lumped parameters, and model deviations are lumped in the values of these properties. Since no model matches reality completely there will always be certain deviations and therefore we will always lump some relevant physical processes in the values of the heat transport properties. This can create apparent dependences of the heat transport properties on certain parameters, as is the case for the dependence on reaction kinetics due to the lumping of the heterogeneous character. If a model is closer to physical reality, there will be less discrepancies and the discrepancies that still occur will be smaller. This urges the choice of a model which is as close to physical reality as possible. Here we will give six reasons why we have chosen the series model:

(1) On intuitive grounds one could argue that the fact that heat transport through the pellet contact points cannot be neglected is confirmed by the fact that the static contribution of the effective heat conductivity of the bed depends on the heat conductivity of the solid phase. Under industrial conditions usually velocities are so high that the value of the static contribution in the effective heat conductivity can be neglected compared to the dynamic contribution, so the effective heat conductivity becomes independent of the contribution of conduction within the solid phase. The series model does not deny heat transport through the solid phase: heat is transported from one pellet to another via the gas phase, but also heat is transferred through the pellet itself. The series model only states that heat transport via the contact spot is negligible. As a matter of fact, since we assume that the temperature of the pellet is uniform we assume an infinitely high conductivity of the pellet. We believe that conduction within the solid phase itself need not be considered because the solid phase is the discontinuous phase. The gas phase is the continuous phase, and therefore radial and axial conduction in the bed is determined to a much greater extent by the so-called effective conduction within the gas phase,

(2) It has also been argued that parallel heat transport cannot be neglected because the stagnant gas interstices near the pellet contact points must be included as part of the solid phase. If this were true the particle surface area should also be corrected to another smaller area really effective for heat transfer from the particle to the gas. We do not see any reason to include those interstices as part of the solid phase whereas they simply consist of gas. Also, this region will contribute to the overall value of the heat transfer coefficient of a single particle in a bed to the surrounding gas,

(3) We did experiments which show that we can describe transient, heterogeneous experimental temperature profiles in a packed bed within a $2 \%$ relative error using a series model. We will report the results of these experiments in the near future. The values found for the heat transport properties from these profiles are in good agreement with values reported in the literature for entirely different systems, e.g. steady-state systems,

(4) We have measured local values for the pellet heat transfer coefficient, also assuming that for a pellet inside a packed bed we have heat transfer from the pellet to the gas only and that there is no direct heat transport from this pellet to its neighbouring pellets, i.e. there also a series model is assumed, now on a pellet scale. These results will also be presented in the near future. Values for the pellet heat transfer coefficient obtained with this model are in excellent agreement with data reported in the literature, again despite the entirely different systems used,

(5) We measured catalyst and gas temperatures inside a packed bed where an exothermic reaction was carried out and found that two neighbouring pellets could easily differ, e.g. $3^{\circ} \mathrm{C}$ in temperature, due to the different catalyst content of the pellets, but the gas flowing around those pellets did not have a sudden jump in temperature. The temperature difference between pellet and gas was, for example, $3^{\circ} \mathrm{C}$ for the cold pellet and $6^{\circ} \mathrm{C}$ for the hot pellet,

(6) Calculations by Bauer (1977) show that the contact spot area between pellets inside a packed bed is so small that the contribution of this contact spot to the static contribution of the effective heat conductivity of the bed is absolutely negligible.

Because of the arguments given above we feel convinced that the series model is much closer to physical reality than the parallel model. 